\title{
Dynamic Modeling and Optimal Control for Complex Systems with Statistical Trajectory
}

\author{
Lingli Guo, ${ }^{1,2}{\text { Zhengguang Xu, }{ }^{1} \text { and Ye Wang }}^{1}$ \\ ${ }^{1}$ School of Automation, University of Science \& Technology Beijing, Beijing 100083, China \\ ${ }^{2}$ Department of Mathematics, Changzhi College, Changzhi, Shanxi 046011, China
}

Correspondence should be addressed to Lingli Guo; guolinglilyw@aliyun.com

Received 29 June 2014; Revised 31 August 2014; Accepted 31 August 2014

Academic Editor: Qingang Xiong

Copyright (c) 2015 Lingli Guo et al. This is an open access article distributed under the Creative Commons Attribution License, which permits unrestricted use, distribution, and reproduction in any medium, provided the original work is properly cited.

\begin{abstract}
This paper is concerned with the problem of the working state class dynamic modeling and control of the complex system with statistical trajectory. Firstly, a novel discrete-time nonlinear working state class dynamic mathematical model of sintering machine is constructed by $K$-means cluster method for the local data, which overcomes difficulties of modeling by mechanism and describes the class change against the point movement. Then, based on the working state class dynamic modeling, the optimal control method which is significant to achieve trajectory tracking for sintering machine is developed by constructing the quadratic performance indicator. More importantly, the method facilitates control process realization from one class to another. Finally, the simulation is provided to illustrate the effectiveness of the proposed method.
\end{abstract}

\section{Introduction}

In control engineering, many techniques for designing a controller, either mathematical analysis or numerical calculations [1-4], are based on the mathematical model of the system. However, if the system is nonlinear or the model of the system is not clearly defined, it is a difficult task to design a controller that satisfies the system requirements. For example, there are complex physical and chemical transformations in sintering machine so that they are difficult to model by the physical nature.

However, we are inspired by workers keeping watch on the fire who recognize the working state by observing the flame type to adjust the firing temperature. To some extent, parameters of the working state such as bellows temperature and bellows pressure which can be measured reflect flame type or working state; it is quite natural to inquire whether we imitate the whole manual control to automate control in order to avoid artificial factors. Dynamic model of the working state classes was built via dividing these parameters values into interval number [5-7]. Predictive control model was established by the class centre value representing the whole class points [8-11]. While these models describe the dynamic moving of flame type or working state class, the class representation is still thought to be significant challenge for working state computation.

In this paper, we show that cell structured state spaces modeling gives us indeed a new possibility. In particular, we present controlled autoregressive moving average (CARMA) modeling method of the dynamics working state class for sintering machine, which is established by dividing state space into cells via $K$-means [9] clustering algorithm. Based on this model, the dynamic programming optimal control strategy is employed to design controller for every initial cell. In spite of optimal control theory based on cell mapping and its various areas of application in engineering, traditional methods [3-12] are known model, and the control table is obtained by cell mapping training. In [13], although the model is unknown, the order is known, and the system dynamic and control table is gained by learning signal.

In this paper, we proposed the modeling method that the system working class dynamic behavior is completely recognized by identifying the system cell dynamics. This method described in greater detail is in Section 2. In [1417], the pattern recognition is static process. Therefore, the dynamics of the system are then described in the form 
TABLE 1: The partial data in parameter space.

\begin{tabular}{lccccc}
\hline WBT20 & WBT21 & WBT22 & EBT20 & EBT21 & EBT22 \\
\hline 419 & 431 & 306 & 401 & 382 & 352 \\
321 & 425 & 329 & 403 & 394 & 353 \\
440 & 449 & 324 & 410 & 387 & 361 \\
371 & 399 & 262 & 418 & 383 & 352 \\
442 & 475 & 290 & 382 & 388 & 382 \\
492 & 452 & 309 & 338 & 387 & 283 \\
401 & 370 & 366 & 437 & 456 & 297 \\
411 & 378 & 357 & 432 & 441 & 374 \\
398 & 384 & 364 & 421 & 434 & 379 \\
421 & 394 & 394 & 424 & 429 & 392 \\
421 & 392 & 384 & 411 & 434 & 369 \\
400 & 374 & 365 & 411 & 432 & 374 \\
435 & 408 & 385 & 380 & 400 & 300 \\
420 & 404 & 375 & 390 & 421 & 270 \\
400 & 409 & 378 & 348 & 430 & 268 \\
415 & 409 & 373 & 350 & 415 & 264 \\
390 & 378 & 370 & 408 & 438 & 268 \\
390 & 390 & 364 & 420 & 430 & 310 \\
433 & 391 & 369 & 454 & 469 & 306 \\
$\ldots$ & $\ldots$ & $\ldots$ & $\ldots$ & $\ldots$ & $\ldots$ \\
\hline
\end{tabular}

of mappings from one cell onto another in this paper. In addition, most of the cell division principles are based on the experience [13, 18-22]. Here, we present the cell division by $K$-means cluster method. The initial category and category center of system working state randomly are selected until the JC [3] value tends to make steady. Meanwhile, combining with the experience, the best clustering result finally is decided. Every cell represents a working state class, so cellto-cell mapping describes the working state class dynamics. References [23-26] involved dynamic system simulation.

The optimal control object is the systems that enter into the target cell representing the best working state from any initial cell with the minimum cost function. Section 2 contains the constructing process of the model. The attractiveness of the method lies in eliminating the division error at the experience level. Section 3 is devoted to introducing the dynamical planning optimal control strategy. Example and simulation of applying the extended method to sinter machine control are finished in Section 4. Section 5 concludes the paper. Emphasis is placed on the construction of dynamic working state model by which the class shift with tracking statistical motion trajectory is evaluated.

\section{Problem Statement and Dynamic Description of the Sintering Machine Working State Class}

In this section, we construct the dynamic model of the working state class for the sintering machine through data driven approach. As it is known, there are complex physical and chemical changes so that the mechanism modeling is very difficult. Meanwhile, the operating mode is only estimated
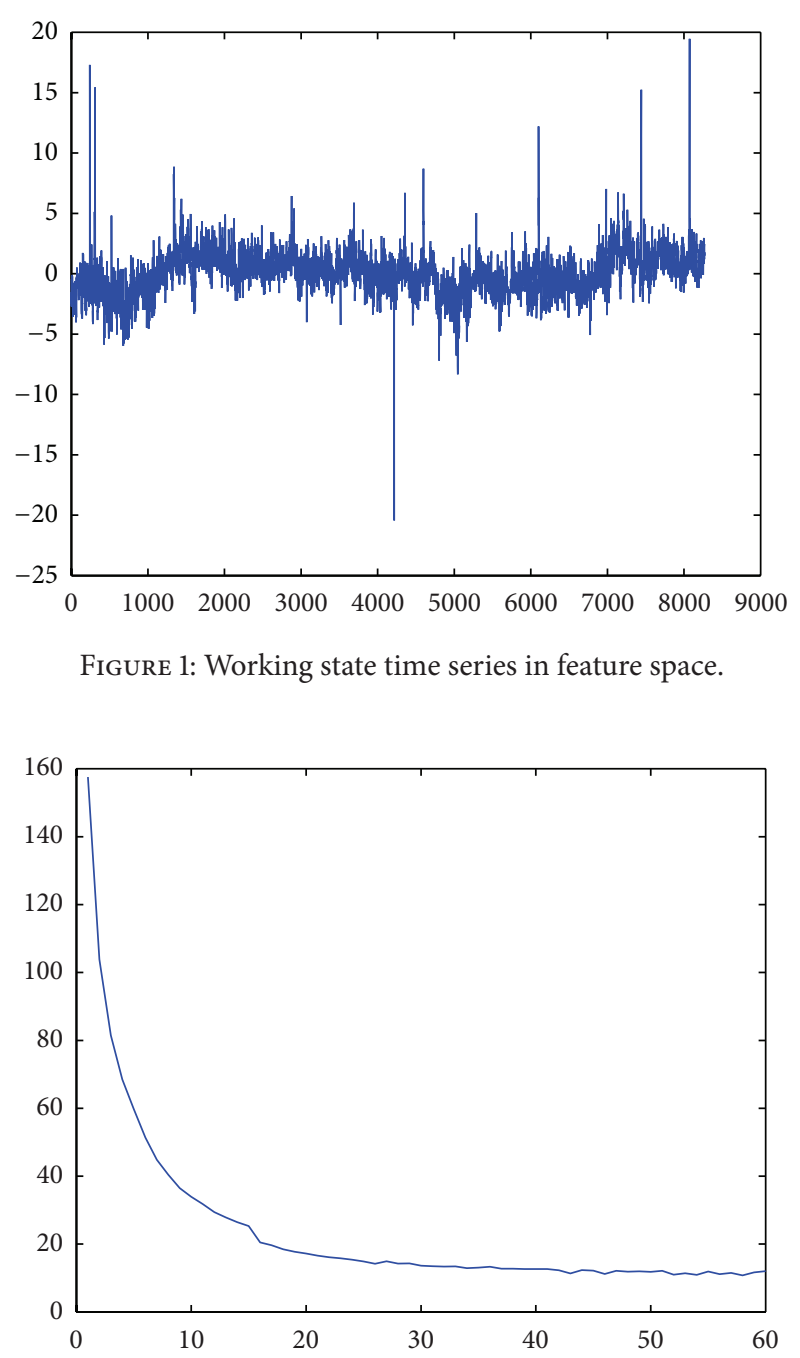

FIGURE 2: JC values change with category numbers.

according to the set of some approximate variables values such as dynamic west bellows temperature and pressure and east bellows temperature and pressure. Therefore, the first step is clustering dynamic variables values. The data must be standardized before clustering. The part of sample data is shown in Table 1, where the west bellows temperature is simplified as WBT and east bellows temperature is EBT.

The standardized data sequence is in Figure 1.

By $\mathrm{K}$-means clustering and JC rules (Figure 2), we find the best clustering number is 17 .

The total clustering centers are shown in Table 2.

For describing the working state class trajectory, the second step is obtaining every instant mode by the clustering centre instead of each element belonging to this class in order to build CARMA model. Firing temperature is the input of the model. Clustering centre is the model output. The order (Figure 3 ) and delay (Figure 4 ) of the model are decided by the inflection point of residual sum function of squares.

The model parameter is estimated by least square method. Then, the dynamic mode model is tested (Figure 5) by residual independence algorithm and statistical $F$-test. 


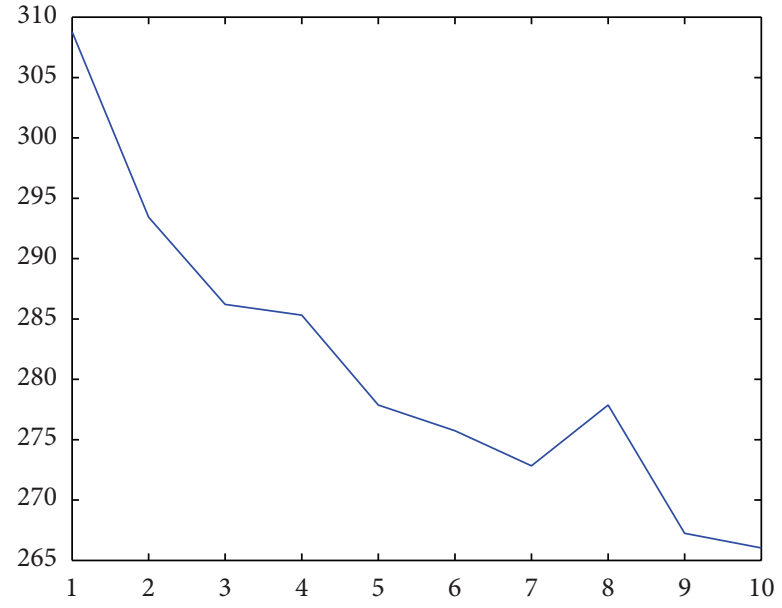

FIGURE 3: Residual sum function of squares change with the order.

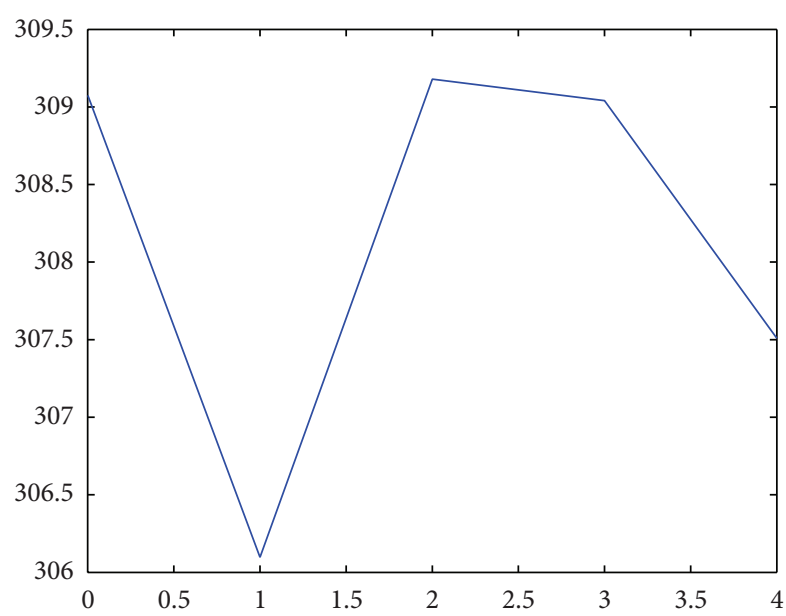

FIGURE 4: Residual sum function of squares change with the delay.

Using the statistical $F$-test method, we set the parameter $\alpha=5 \%$ and gain $F_{\alpha}(\infty)=3$. Table 3 is from the formula $F=\left(\left(J\left(n_{1}\right)-J\left(n_{2}\right)\right) / 2\left(n_{2}-n_{1}\right)\right) \times\left(\left(L-2 n_{2}\right) / J\left(n_{2}\right)\right)$.

Finally, the system model can be described as

$$
\begin{gathered}
\tilde{y}(k)-0.4296 y(k-1)-0.1706 y(k-2)-0.0896 y(k-3) \\
=0.1362 u(k-1)-0.0443 u(k-2)-0.0068 u(k-3), \\
y(k)=F(\tilde{y}(k)),
\end{gathered}
$$

where $\tilde{y}(k)$ is the initial output of the system at $k$ instant, $y(k)$ is corresponding class centre, $F$ is classifier, and $u(k)$ is the input.

\section{Dynamical Optimal Controller Design}

The controller is designed to guide the sintering machine working state class to follow a predefined class trajectory. To
TABLE 2: Every class number and class centre.

\begin{tabular}{lc}
\hline Class number & Class centre \\
\hline 1 & -20.4331 \\
2 & -4.9354 \\
3 & -3.3971 \\
4 & -2.4656 \\
5 & -1.7454 \\
6 & -1.1826 \\
7 & -0.6749 \\
8 & -0.2130 \\
9 & 0.2429 \\
10 & 0.6650 \\
11 & 1.1314 \\
12 & 1.6472 \\
13 & 2.2207 \\
14 & 2.9349 \\
15 & 4.0309 \\
16 & 6.5470 \\
17 & 14.1273 \\
\hline
\end{tabular}

TABLE 3: Every class number and class centre.

\begin{tabular}{lcc}
\hline The order $n$ & Residual sum of squares $J$ & $F$-value \\
\hline 1 & 314.5495 & \\
2 & 296.1067 & 13.8894 \\
3 & 289.0520 & 5.4182 \\
4 & 287.7628 & $0.9901<3$ \\
5 & 279.3846 & 0.1452 \\
\hline
\end{tabular}

obtain the control of the system, we assume the state variable is

$$
\begin{gathered}
x_{1}(k+1)=x_{2}(k)+h_{2} u(k), \\
x_{2}(k+1)=x_{3}(k)+h_{1} u(k), \\
x_{3}(k+1)=-a_{0} x_{1}(k)-a_{1} x_{2}(k)-a_{2} x_{3}(k)+h_{0} u(k) .
\end{gathered}
$$

The output is expressed as

$$
y(k)=x_{1}(k)+h_{3} u(k),
$$

where $h_{3}=b_{3}, h_{2}=b_{2}-a_{2} h_{3}, h_{1}=b_{1}-a_{1} h_{2}$, and $h_{0}=$ $b_{0}-a_{0} h_{3}-a_{1} h_{2}-a_{2} h_{1}, a_{i}, b_{i}$, and $h_{i}$ are given by the following model:

$$
\begin{aligned}
& y(k)+a_{2} y(k-1)+a_{1} y(k-2)+a_{0} y(k-3) \\
& =b_{3} u(k)+b_{2} u(k-1)+b_{1} u(k-2)+b_{0} u(k-3) .
\end{aligned}
$$




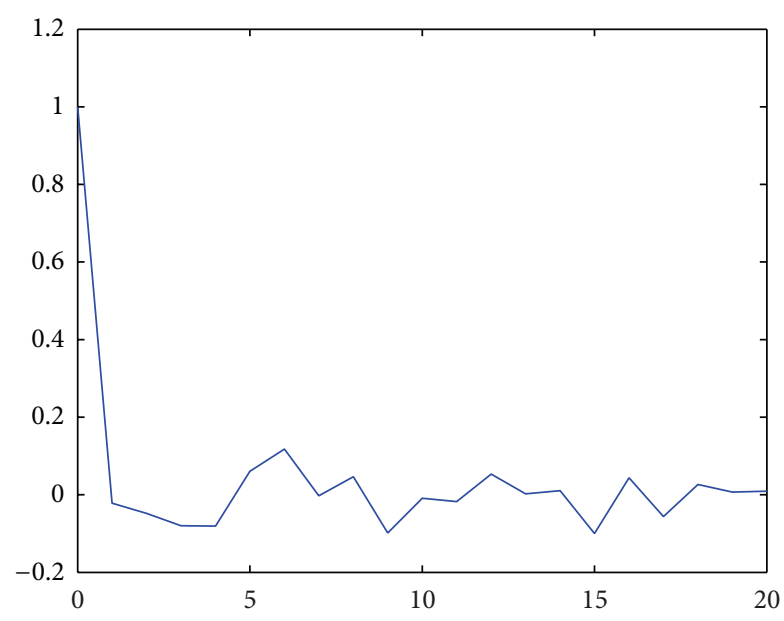

Figure 5: Residual independence series test.

In order to implement the control for the system, the discrete-time state space model can be obtained as follows:

$$
\begin{aligned}
& {\left[\begin{array}{l}
x_{1}(k+1) \\
x_{2}(k+1) \\
x_{3}(k+1)
\end{array}\right]=\left[\begin{array}{ccc}
0 & 1 & 0 \\
0 & 0 & 1 \\
0.0896 & 0.1706 & 0.4296
\end{array}\right]\left[\begin{array}{l}
x_{1}(k) \\
x_{2}(k) \\
x_{3}(k)
\end{array}\right]} \\
& +\left[\begin{array}{c}
0.1362 \\
-0.0211 \\
-0.0073
\end{array}\right] u(k) \\
& y(k)=\left[\begin{array}{lll}
1 & 0 & 0
\end{array}\right]\left[\begin{array}{l}
x_{1}(k) \\
x_{2}(k) \\
x_{3}(k)
\end{array}\right],
\end{aligned}
$$

and (5) can be simplified as

$$
\begin{gathered}
X(t+1)=A X(t)+B U(t), \\
y(t)=C X(t) .
\end{gathered}
$$

Combining the expert knowledge with the above system analysis, the steady-state operating mode centre for reference can be set as

$$
r_{0}=0.6650 .
$$

Now, the steady-state state space model is obtained by (6), (7), and (8):

$$
\begin{gathered}
r_{0}=C X_{0}, \\
X_{0}=A X_{0}+B u_{0} .
\end{gathered}
$$

Therefore, we can get the steady-state part of the control variable and state variable by (10):

$$
\begin{gathered}
u_{0}=\left(C(I-A)^{-1} B\right)^{-1} r_{0}, \\
X_{0}=(I-A)^{-1} B u_{0} .
\end{gathered}
$$

The associated cost function is defined by

$$
J=\sum_{k=0}^{\infty}\left[x(k)^{T} Q x(k)+u(k)^{T} R u(k)\right],
$$

where $x(k)=X(k)-X_{0}, x(k) \in R^{n}$, is the state of the system at instant $k \in N^{+}=\{0,1,2, \ldots\} ; u(k)=U(k)-u_{0}, u(k) \in R^{m}$, is input of the control; $Q \in R^{n * n}$ and $R \in R^{m * m}$ are the real symmetric positive definite weighting matrix. In this paper, we assume that all the states are measurable and available. The controller object is finding the controller series $\{\hat{u}(k), k=$ $0,1, \ldots, N-1\}$ so that the cost function (12) is the minimum.

And (12) can be transformed into

$$
\begin{aligned}
J(x(k))= & x^{T}(k) \mathrm{Q} x(k)+u^{T}(k) R u(k) \\
& +\sum_{n=k+1}^{\infty}\left(x^{T}(n) Q x(n)+u^{T}(k) R u(k)\right) \\
= & x^{T}(k) Q x(k)+u^{T}(k) R u(k)+\widehat{J}(x(k+1)),
\end{aligned}
$$

where $J\left(x_{0}\right)=0$ is required as the boundary condition. Therefore, $J\left(x_{k}\right)$ becomes a Lyapunov functional. Based on Bellman's optimality principle, the cost functional $J\left(x_{k}\right)$ becomes invariant and satisfies the discrete-time Hamiltonian for the infinite horizon optimization case, and it becomes

$$
\begin{aligned}
\widehat{J}(x(k)) & \\
& =\min _{u(k)}\left\{x^{T}(k) Q x(k)+u^{T}(k) R u(k)+\widehat{J}(x(k+1))\right\} .
\end{aligned}
$$

We guess the solution of Bellman's equation can be expressed as

$$
\widehat{J}(x(k))=x^{T}(k) G(k) x(k),
$$

where $G(k) \in R^{n * n}$ is the real symmetric variable matrix.

Substituting (14) into (15) yields

$$
\begin{aligned}
\widehat{J}(x(k))=x^{T}(k) G(k) x(k) \\
=\min _{u(k)}\left\{x^{T}(k) Q x(k)+u^{T}(k) R u(k)\right. \\
\left.\quad+x^{T}(k+1) Q x(k+1)\right\} .
\end{aligned}
$$

Combining (15) and (6), it follows that

$$
\begin{aligned}
& \widehat{J}(x(k)) \\
& =x^{T}(k) G(k) x(k) \\
& =\min _{u(k)}\left\{x^{T}(k) Q(k) x(k)+u^{T}(k) R u(k)\right. \\
& \left.\quad+\left(A x(k)+B u(k)^{T}\right) G(k+1)(A x(k)+B u(k))\right\} \\
& =\min _{u(k)}\left\{x ^ { T } ( k ) \left\{Q(k)+A^{T} G(k+1) A\right.\right. \\
& \left.\quad-F^{T}(k)\left(R+B^{T} G(k+1) B\right) F(k)\right\} x(k)
\end{aligned}
$$




$$
\begin{aligned}
& +(u(k)+F(k) x(k))^{T} \\
& \left.\times\left(R+B^{T} G(k+1) B\right)(u(k)+F(k) x(k))\right\},
\end{aligned}
$$

where

$$
F(k) \stackrel{\text { def }}{=}\left(R+B^{T} G(k+1) B\right)^{-1} B G(K+1) A .
$$

In (6), the second part is only related with $u(k)$, and also it is easy to prove that $R(k)+B^{T} G(k+1) B$ is positive define matrix. Therefore, the enough and necessary condition for minimizing the cost function $\widehat{J}$ is

$$
\widehat{u}(k)=-F(k) x(k) .
$$

The optimal control law is obtained.

Substituting (19) into (17) yields

$$
\begin{aligned}
\widehat{J}(x(k))=x^{T}(k) G(k) x(k) & \\
=x^{T}(k)\left\{Q+A^{T} G(k+1) A\right. & \\
& \left.\quad F^{T}(k)\left(R+B^{T} G(k+1) B\right) F(k)\right\} x(k) .
\end{aligned}
$$

Considering (17) exists for every $x(k)$, so

$$
\begin{aligned}
G(k)= & Q+A^{T} G(k+1) A \\
& -F^{T}(k)\left(R+B^{T} G(k+1) B\right) F(k) .
\end{aligned}
$$

Substituting (18) into (21) yields the $G(k)$ meeting the following Riccati equation:

$$
\begin{aligned}
G(k)= & Q+A^{T} G(k+1) A \\
& -A^{T} G(k+1) B\left(R+B^{T} G(k+1) B\right)^{-1} B G(k+1) A,
\end{aligned}
$$

where $G(N)=Q$ is required as the boundary condition.

As discussed above, the discrete-time optimal control problem algorithm can be established as follows.

(i) According to the parameters $A, B, R, Q, S$ of the discrete-time state space model, we build the Riccati equation (22).

(ii) From $G(N)$, by reverse recursive method, calculating the $G(N-1), G(N-2), \ldots, G(1), G(0)$ and then substituting them into (18) and (19) yield $\widehat{u}(k)$.

(iii) Combining $x(k)=X(k)-X_{0}, u(k)=U(k)-u_{0}$ and the initial condition, the optimal control law can be obtained by

$$
U(k)=-F x(k)+F X_{0}+u_{0} .
$$

Similarly, the state trajectory is

$$
X(k+1)=(A-B F(k)) X(k)+B F(k) X_{0}+B u_{0} .
$$

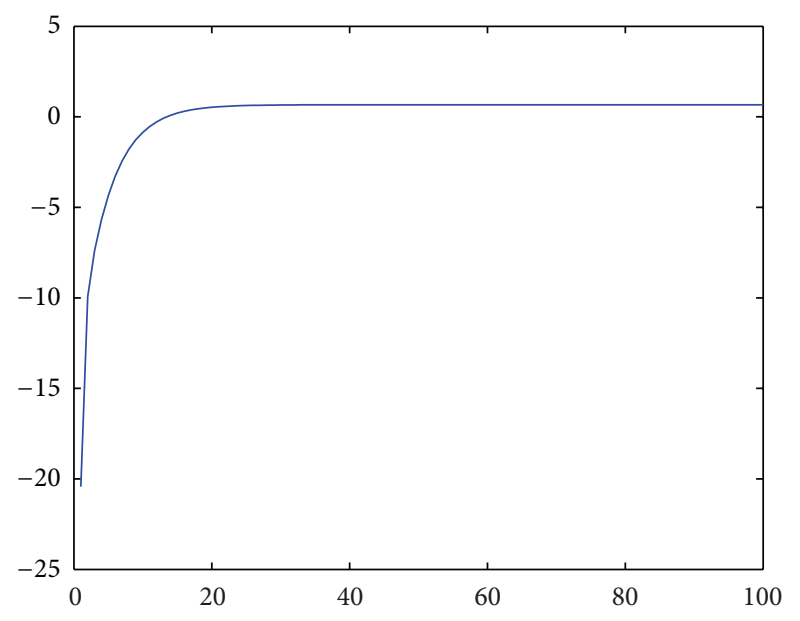

FIGURE 6: Initial system output trajectory.

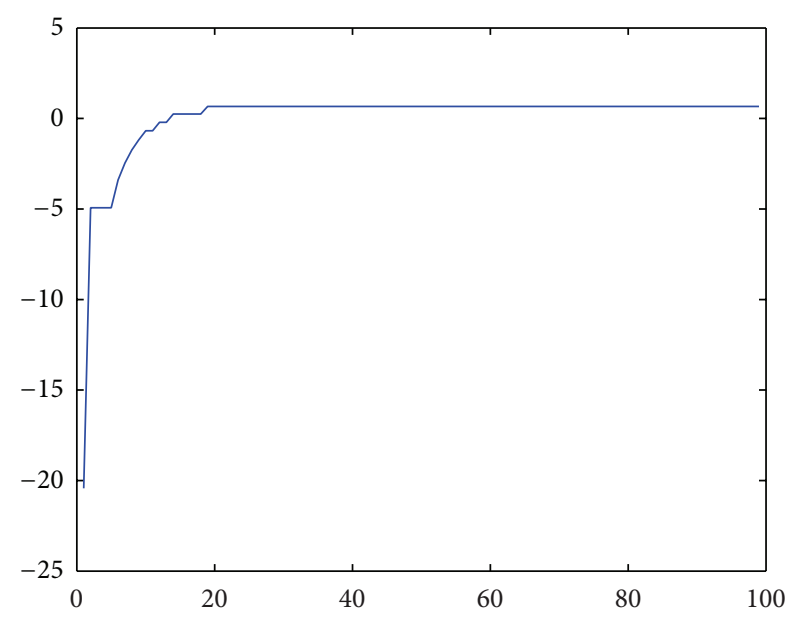

FIGURE 7: Finial system output trajectory.

Substituting (24) into output model yields

$$
y(t)=C X(t)=x_{1}(t) .
$$

Meanwhile, due to the one-to-one mapping of the state space and cell space, we can gain the corresponding the state cell trajectory and output cell trajectory.

However, every cell is possibly the initial cell in sinter machine working state class, so we can obtain a control law for every cell. By (23), the controller is the best so that the cost function is optimal.

The controller design is completed at this point.

\section{Numerical Simulations}

The performances of the proposed optimal controller are illustrated by simulation. The principle of simulation setup based on the experimental schematic is described by Section 3.

(1) The results for the initial state cell $[1,1,1]$ are shown in Figures 6-9.

(2) The results for the initial state cell $[17,17,17]$ are shown in Figures 10-13. 


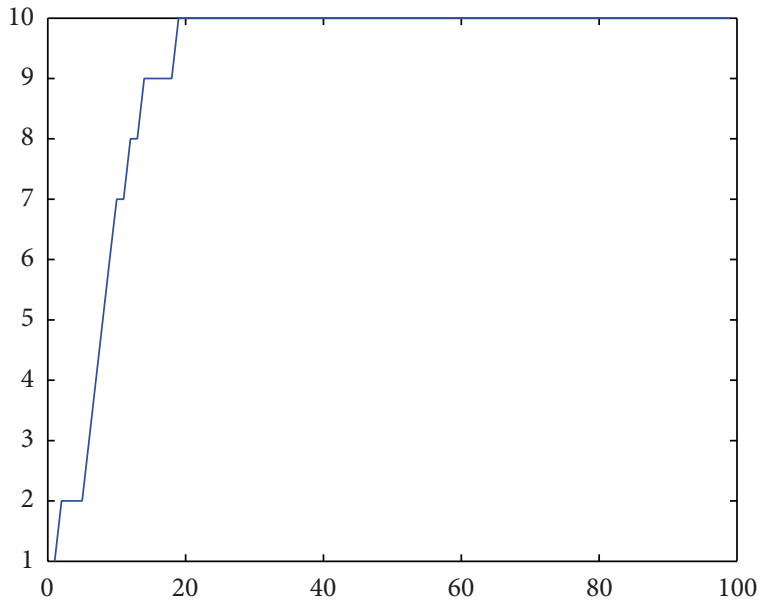

Figure 8: System output cell trajectory.

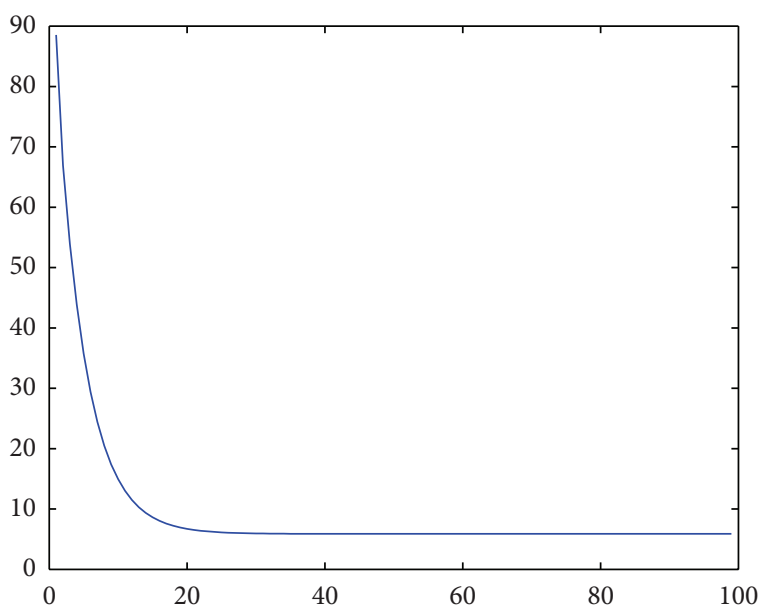

FIGURE 9: System input trajectory.

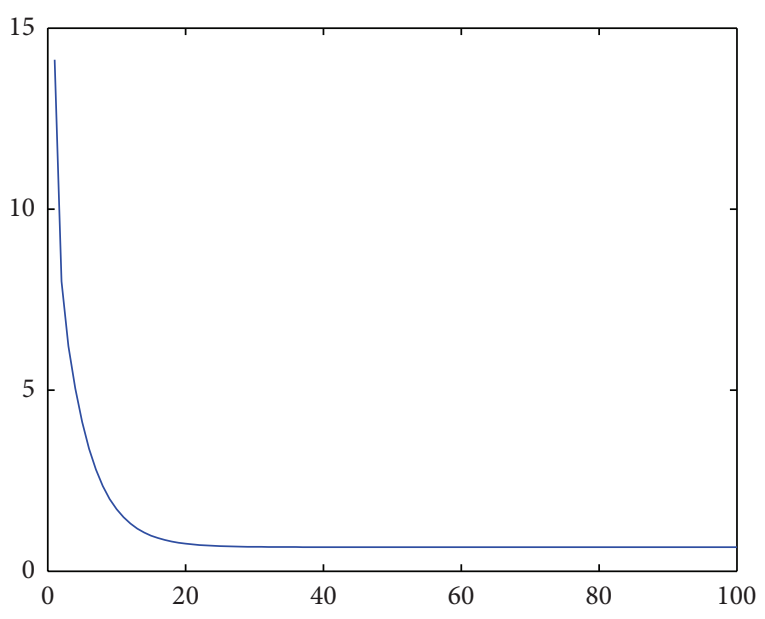

FIGURE 10: Initial system output trajectory.
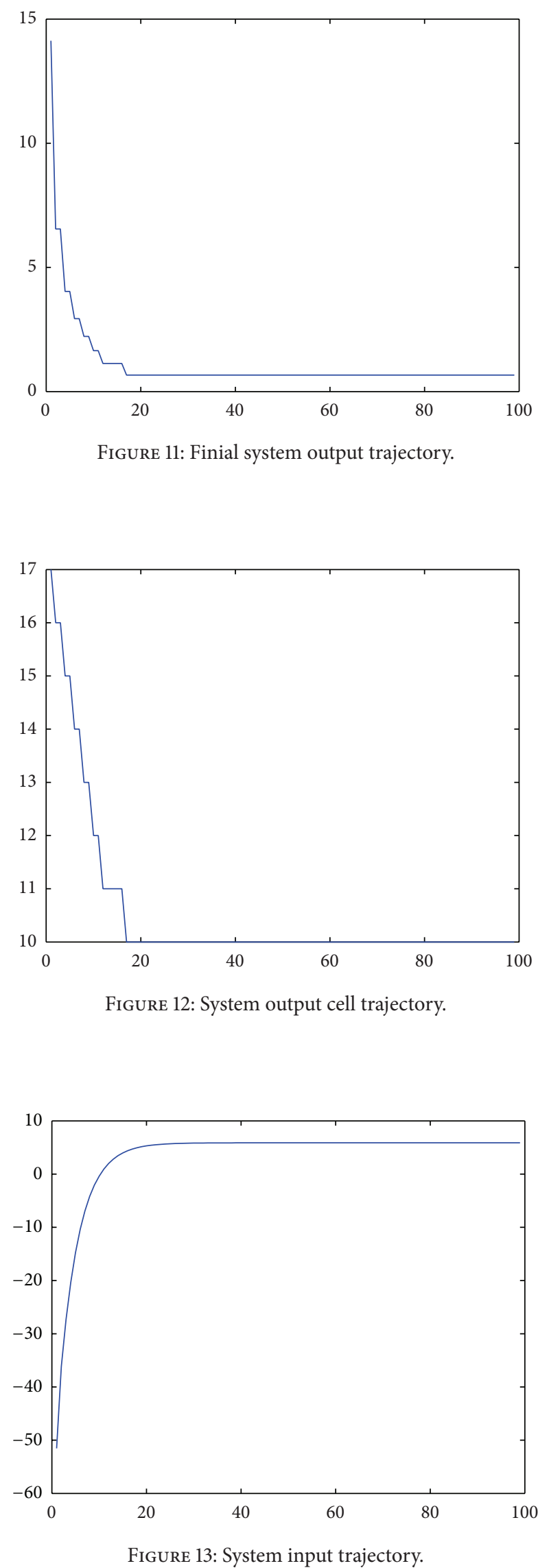
Dynamic tracking of the sintering machine working state class response is shown in Figures 6, 7, 8, 10, 11, and 12. Since the best working state class is the number 9 cell, its state reference is set to the cell centre 0.6650 . From these figures, it is obvious that the optimal controller can realize zero steady-state error and has good dynamic and steady response characteristics.

\section{Conclusion}

The ignition temperature is a key factor affecting the sinter performance; thus its automation control contributes to maintaining good quality. In view of this idea, this paper has proposed a method to model cell dynamics describing the working state class for a class of complex systems with statistical trajectory characteristics. Based on the dynamic programming optimal algorithm, the control law is constructed for each initial cell. The model method and optimal control strategy have important advantages in terms of state classification by cell division and dynamic class description. Their weaknesses (such as the cell boundary and stability) are constantly improved. This unconventional approach to describe the class trajectory is probably the tip of the iceberg in the field of the working state class modeling and control that will emerge in the coming years. A truly multidisciplinary research approach, where all the aspects of such complex dynamic systems have to be considered and fully understood, is required to fully exploit the potential of this new branch of applied science.

\section{Conflict of Interests}

The authors declare that there is no conflict of interests regarding the publication of this paper.

\section{Acknowledgments}

This work was supported by the National Natural Science Foundation of China (Grant no. 61175122) and Key Laboratory of Innovation Method and Decision Management System of Guangdong Province, Guangzhou, Guangdong (Grant no. 2011A060901001-14D). Professor Xu is the first author's tutor for a doctor's degree. His idea that pattern trajectory describes the complex systems moving influences the first author deeply. But he does not point to the specific methods how to characterize the pattern and control the pattern trajectory.

\section{References}

[1] C. S. Hsu, "A discrete method of optimal control based upon the cell state space concept," Journal of Optimization Theory and Applications, vol. 46, no. 4, pp. 547-569, 1985.

[2] L. G. Crespo and J. Q. Sun, "Fixed final time optimal control via simple cell mapping," Nonlinear Dynamics, vol. 31, no. 2, pp. 119-131, 2003.

[3] E. P. Paladini, "A pattern recognition and adaptive approach to quality control," WSEAS Transactions on Systems and Control, vol. 3, no. 7, pp. 627-643, 2008.
[4] L. G. Crespo and J. Q. Sun, "Stochasstic optimal of nonlinear dynamic systems via Bellman's principle and cell mapping," Automatica, vol. 39, no. 12, pp. 2109-2114, 2003.

[5] Z. G. Xu and C. P. Sun, "Moving pattern forecasting using interval T-S fuzzy model," Control and Decision, vol. 27, no. 11, pp. 1699-1710, 2012.

[6] Z. Xu, C. Sun, and J. Wu, "Moving pattern measured by interval number for modeling and control," Control Theory \& Applications, vol. 29, no. 9, pp. 1115-1124, 2012.

[7] Z. Xu and C. Sun, "Moving pattern-based forecasting model of a class of complex dynamical systems," in Proceedings of the 50th IEEE Conference on Decision and Control and European Control Conference (CDC-ECC '11), pp. 4967-4972, Orlando, Fla, USA, December 2011.

[8] Z. Xu and J. Wu, "Pattern recognition: an alternative to dynamics description," in Proceedings of the 51st IEEE Conference on Decision and Control and European Control Conference, pp. 5566-5571, Maui, Hawaii, USA, December 2012.

[9] Z. Xu and J. Wu, "Data-driven pattern moving and generalized predictive control," in Proceedings of the IEEE International Conference on Systems, Man, and Cybernetics (SMC '12), pp. 16041609, Seoul, Republic of Korea, October 2012.

[10] Z.-G. Xu and J.-X. Wu, "Pattern moving trajectory: a new dynamics description method," International Journal of Modelling, Identification and Control, vol. 17, no. 4, pp. 370-379, 2012.

[11] Z. Xu, J. Wu, and S. Qu, "Prediction model based on moving pattern," Journal of Computers, vol. 7, no. 11, pp. 2695-2701, 2012.

[12] Z. Xu, J. Wu, and Y. Wang, "A pattern recognition approach to model a class of complex systems," Journal of Computational Information Systems, vol. 7, no. 3, pp. 4636-4643, 2011.

[13] Y.-Y. Hsu, W.-L. Chen, and E. C. Yeh, "Optimal learning control via cell mapping," International Journal of Systems Science, vol. 28, no. 3, pp. 277-282, 1997.

[14] D. Elliman, "Pattern recognition and financial time-series," International Journal of Intelligent Systems in Accounting, Finance and Management, vol. 14, no. 3, pp. 99-115, 2006.

[15] M. D'Acunto and O. Salvetti, "Pattern recognition methods for thermal drift correction in Atomic Force Microscopy imaging," Pattern Recognition and Image Analysis, vol. 21, no. 1, pp. 9-19, 2011.

[16] V. Mitra, H. Nam, C. Y. Espy-Wilson, E. Saltzman, and L. Goldstein, "Articulatory information for noise robust speech recognition," IEEE Transactions on Audio, Speech and Language Processing, vol. 19, no. 7, pp. 1913-1924, 2011.

[17] Q. P. He and J. Wang, "Large-scale semiconductor process fault detection using a fast pattern recognition-based method," IEEE Transactions on Semiconductor Manufacturing, vol. 23, no. 2, pp. 194-200, 2010.

[18] C. S. Hsu, "A theory of cell-to-cell mapping dynamical systems", American Society of Mechanical Engineers, vol. 47, no. 4, pp. 931939, 1980.

[19] F.-Y. Wang and P. J. A. Lever, "A cell mapping method for general optimum trajectory planning of multiple robotic arms," Robotics and Autonomous Systems, vol. 12, no. 1-2, pp. 15-27, 1994.

[20] C. S. Hsu, "Global analysis by cell mapping," International Journal of Bifurcation and Chaos in Applied Sciences and Engineering, vol. 2, no. 4, pp. 727-771, 1992.

[21] D.-B. Zhao, D.-R. Liu, and J.-Q. Yi, "An overview on the adaptive dynamic programming based urban city traffic signal optimal control," Acta Automatica Sinica, vol. 35, no. 6, pp. 676-681, 2009. 
[22] C. S. Hsu, Cell-to-Cell Mapping:A Method of Global Analysis for Nonlinear Systems, Springer, New York, NY, USA, 1987.

[23] J. Zhang, X. Huang, Y. Yue, J. Wang, and X. Wang, "Dynamic response of grapheme to thermal impulse," Physical Review B, vol. 84, no. 23, Article ID 235416, 12 pages, 2011.

[24] J. Zhang, X. Wang, and H. Xie, "Co-existing heat currents in opposite directions in graphene nanoribbons," Physics Letters A, vol. 377, no. 41, pp. 2970-2978, 2013.

[25] Q. Xiong, B. Li, and J. Xu, "GPU-accelerated adaptive particle splitting and merging in SPH," Computer Physics Communications, vol. 184, no. 7, pp. 1701-1707, 2013.

[26] Q. Xiong, L. Deng, W. Wang, and W. Ge, "SPH method for twofluid modeling of particle-fluid fluidization," Chemical Engineering Science, vol. 66, no. 9, pp. 1859-1865, 2011. 


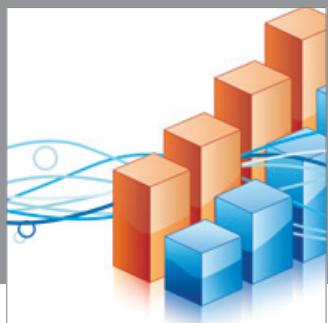

Advances in

Operations Research

mansans

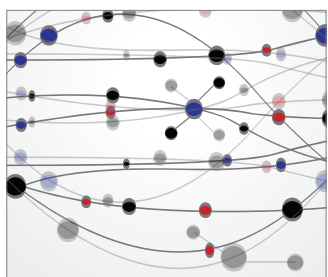

The Scientific World Journal
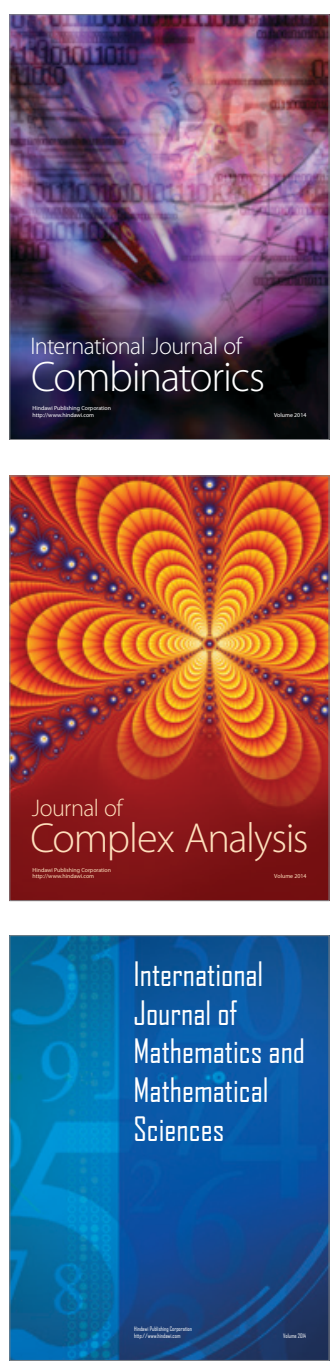
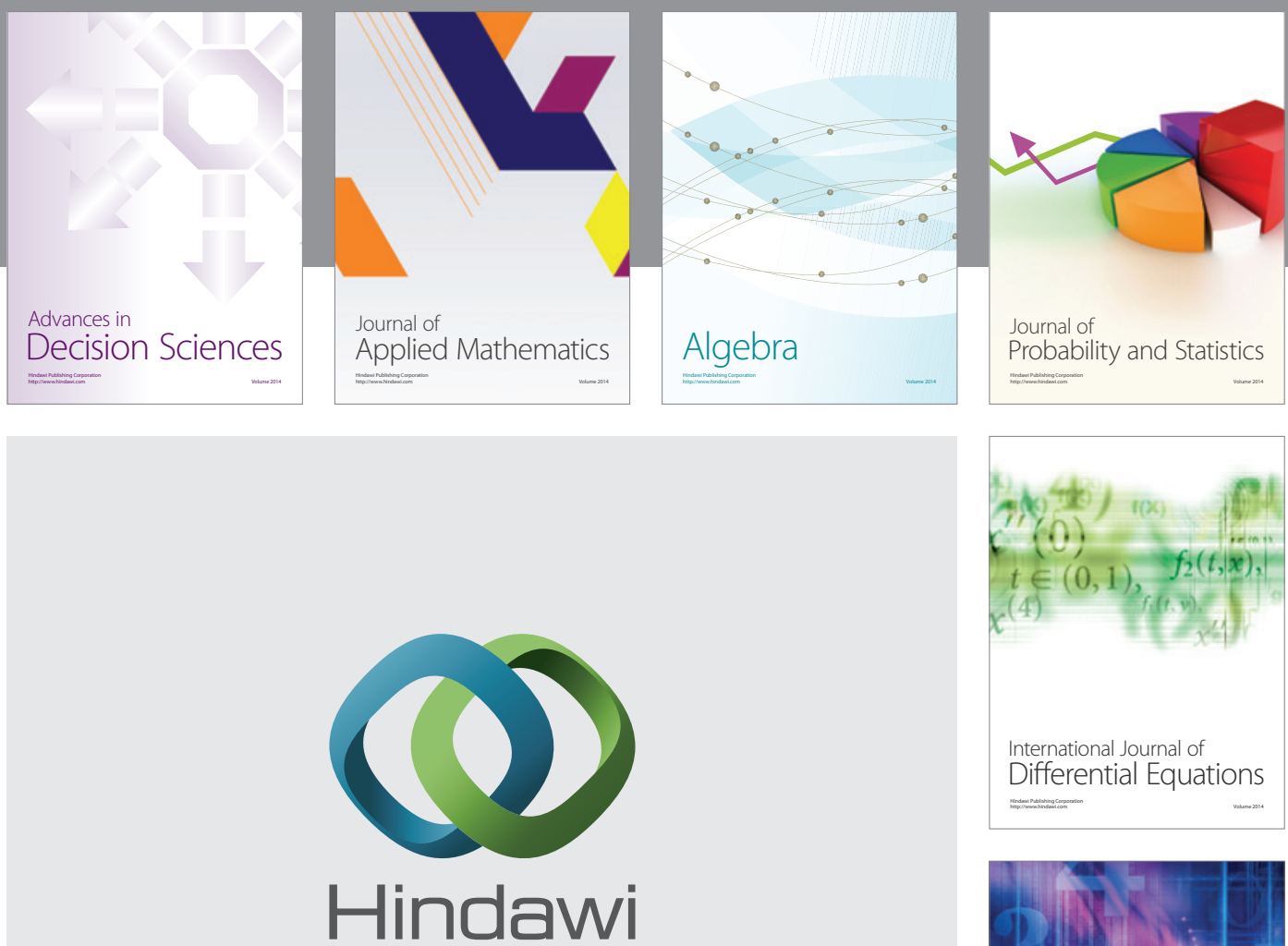

Submit your manuscripts at http://www.hindawi.com
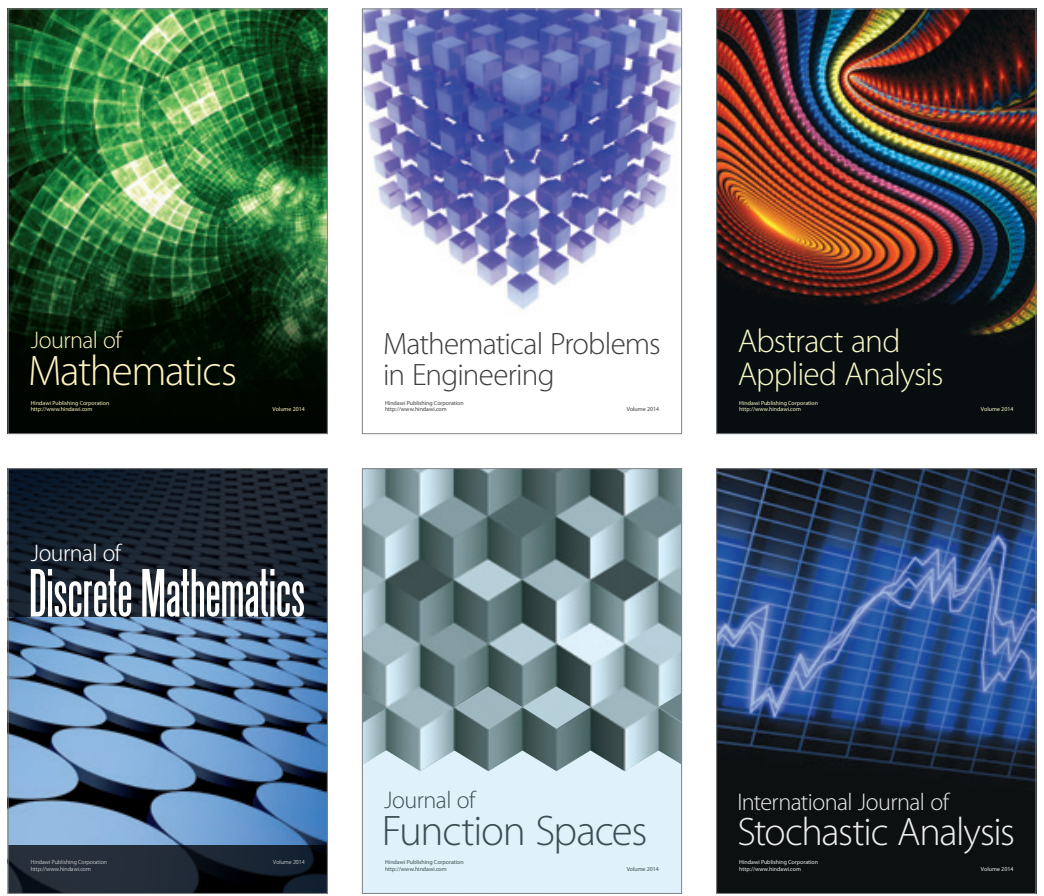

Journal of

Function Spaces

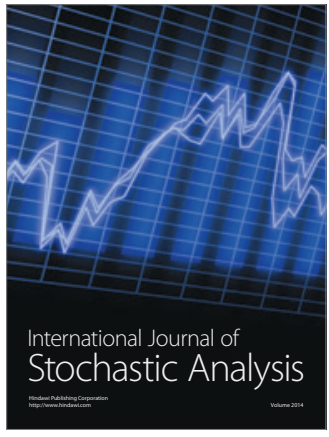

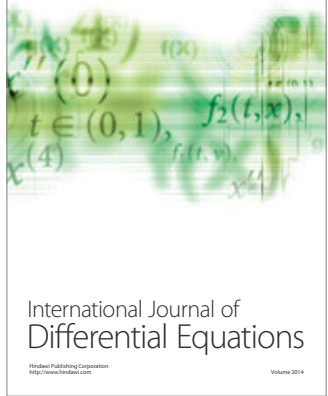
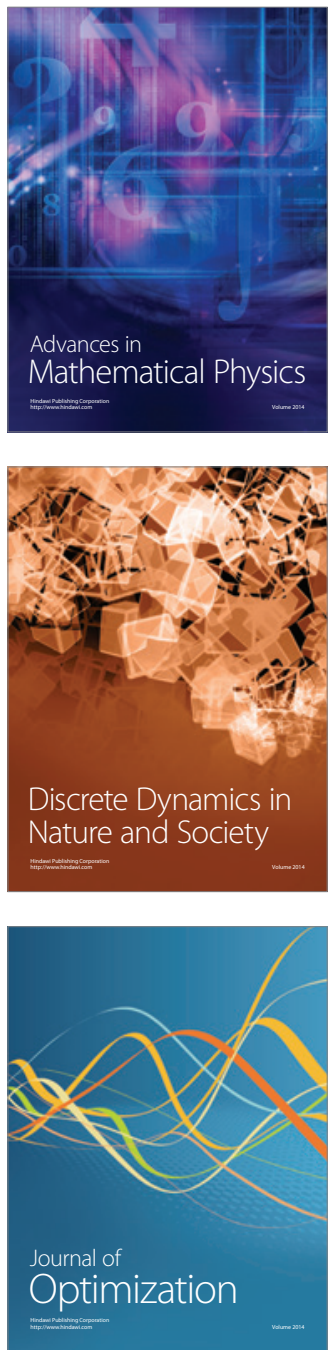